\title{
Validation of Automated Dilution and Transfer Methods for Rapid Assessment of Functional Immune Responses to Meningococcal Vaccines
}

\author{
Angela M. Payne*, Xu Liu, Michael J. Caulfield, Jon H. Heinrichs \\ Vaccines Basic Research, Merck Sharp and Dohme Corp., West Point, USA. \\ Email: *angela_payne2@merck.com
}

Received May $8^{\text {th }}, 2013$; revised June $5^{\text {th }}, 2013$; accepted June $18^{\text {th }}, 2013$

Copyright (C) 2013 Angela M. Payne et al. This is an open access article distributed under the Creative Commons Attribution License, which permits unrestricted use, distribution, and reproduction in any medium, provided the original work is properly cited.

\begin{abstract}
A high-throughput Serum Bactericidal Assay (SBA) was developed to monitor the functional antibody responses to capsular polysaccharide antigens from multiple serotypes of Neisseria meningitidis. This assay measures the ability of an antibody, aided by complement, to mediate killing of bacteria. Functional assays of this type are increasingly used in the quality control arena as potency release tests. Consequently, there has been an enhanced requirement for reproducibility in the performance of this type of assay. Assay validation is, therefore, important to facilitate understanding of the significance of values obtained, and to enable appropriate and informed use of the assay. This study involved the evaluation of the high-throughput serum bactericidal assay including the effects of different dilution and transfer techniques on assay performance. The results presented here demonstrate the repeatability, and the precision and ruggedness of the assay.
\end{abstract}

Keywords: Serum Bactericidal Assay; SBA; Neisseria meningitides; Automation; Repeatability

\section{Introduction}

The accurate enumeration of viable bacteria is critical in the application of antibiotic screening assays, toxicology testing, and serological assays for functional antibodies. Serological assays to measure functional antibodies have changed little over the last 50 years. A standard assay for measuring serum bactericidal activity (SBA) [1] is well accepted as a correlate for protection for which the read-out is bacterial colony growth on agar plates. More recently, a four-valent meningococcal vaccine (Menactra ${ }^{\circledR}$, Sanofi-Pasteur, Swiftwater, PA) was licensed based upon this assay [2]. An alternative method [3], consisting of growing bacteria on agar medium in individual wells of a 96-well plate, has reduced the number of plates required, but the resulting colonies must still be counted manually. These standard agar-based assays are laborintensive and have limited throughput. More recently, a more efficient, faster, high-throughput assay was developed. The method allows for the growth, staining, and counting of bacterial colonies in 96-well filter plates, and has been applied to the measurement of SBA in human

${ }^{*}$ Corresponding author. sera [4]. The method uses the rapid counting of stained colonies with an imaging system originally designed to count spots resulting from the production of cytokines by activated $T$ cells (ELISPOTs). Here we present further improvements to this SBA technique using automated methods for dilution and transfer of test samples. Furthermore, we report the validation of automated dilution and transfer methods in the application of this method to the measurement of functional antibodies.

In general, serological assays are validated using sera from individuals with known responses to the pathogen of interest. Typically, control sera from age-matched individuals are used; due to limitations in availability of sera from pediatric populations, validation of SBA is frequently performed using adult sera. However, adults often harbor pre-existing antibodies with bactericidal activity against Neisseria meningitidis resulting from environmental exposure to pathogens or commensal organisms that share cross-reactive epitopes. This is particularly problematic for serogroup $\mathrm{B}$, to which most adults have antibodies. An exception is serogroup A, which is rare in the United States. The validation of the assay described here was performed for serogroups A 
and $\mathrm{B}$.

\section{Materials and Methods}

\subsection{Bacteria}

N. meningitidis serogroup A (strain number 13077) and serogroup B (strain number 13090) were obtained from the ATCC (Manassas, VA) and were grown as previously described [4].

\subsection{Complement}

Normally, guinea pig sera are the preferred source of complement. However, baby rabbit sera are readily available and have shown to produce similar results. Therefore, pooled baby rabbit sera obtained from Pel-Freez, Inc. (Rogers, AR) served as the complement source.

\subsection{Meningococcal Sera}

Negative control sera from normal adult volunteers without known exposure to $N$. meningitidis serogroups A or B, previously tested and shown not to have titers, were obtained from Biological Specialty, (Hatfield, PA). Serogroup A positive sera were obtained from donors vaccinated with a licensed tetravalent (A, C, Y and W-135) polysaccharide vaccine (Menomune $\AA$, Sanofi Pasteur). Serogroup B positive sera were obtained from human adult volunteers, previously tested and shown to produce high titers to serogroup B. Test sera were serially diluted in assay buffer by either manual dilution using a multichannel pipetter (Labsystems, Thermo Scientific, Waltham, MA) or by automated dilution using a Serial Mate Multichannel System (Apogent Discoveries, Matrix Technologies Corporation, Thermo Scientific). Serum bactericidal activity was determined as previously described [4].

\section{Results}

Serum samples were tested against bacteria of either serogroup A or B meningococci. All sera were evaluated by two-fold serial dilution from a 1:8 to 1:1024 and tested in duplicate in adjacent wells. SBA titers were determined as the reciprocal of the greatest serum dilution resulting in 50\% killing, when compared to the mean of the colony counts in the complement control wells. Samples with serum bactericidal activity titers $<8$ were reported as 4 and regarded as negative. The percent killing was calculated according to the following formula:

$\%$ Killing $=\left(1-\frac{\mathrm{CFU} / \text { well }}{\mathrm{CFU} \text { mean of complement control }}\right) \times 100 \%$

The assay validation was designed to evaluate possible sources of variability. To examine the reproducibility of the methods, data were collected by two analysts using the same equipment and sub-sampling from the same stock samples. The precision and ruggedness of the assay were evaluated by analyzing the reproducibility from multiple assays and different analyses of the same sample. Each serum sample was tested three times in parallel. Two dilution methods, manual (multi-channel pipet) and automated (Serial Mate), and two transfer methods, manual (multi-channel pipet) and automated (Rapid Plate, Qiagen Inc., Valencia, CA) were used. Test sera from five recipients of vaccine as well as sera from individuals that had not been vaccinated were included in the validation.

Repeatability of the assay was evaluated from data obtained from multiple sub-samplings of a homogeneous sample under the same conditions by two analysts, and analyzed separately. The data analysis and the results represent the titers expressed as Log 2 of the reciprocal of the dilution. As expected, the results obtained for serogroup A demonstrated that all sera from vaccinated individuals were positive (titers $\geq 8$ ), and sera from individuals not known to have prior exposure to this serogroup were negative (titers $<8$ ) (Figure 1(a)). Additionally, all samples evaluated for serogroup B resulted in SBA titers $\geq 8$ (data not shown), confirming our expectation that most adults possess detectable bactericidal activity against this serogroup.

The overall precision and ruggedness of the assay was evaluated by comparing day-to-day variability of a negative (Donor 1) and a positive (Donor 9) sera. Data obtained by two analysts using different dilution and transfer methods (manual vs. automated) indicated all samples showed variability to be \pm 1 dilution step, regardless of manual or automated methods of dilution or transfer. When greater variability occurred, it was consistently associated with analyst-to-analyst over day-to-day variability (Figure 1(b)). Samples analyzed for serogroup B produced similar results (data not shown).

Additionally, the method-to-method variability of the assay is represented by data generated by two analysts applying two different dilution and transfer methods for serogroup A (Figure 2(a), (b)). Samples evaluated for serogroup B produced similar results (data not shown). Results indicate a higher variability between analysts when manual dilution or manual transfer methods were used, although differences were small.

Further, the specificity of antisera for the serogroup tested was examined by adding an excess of free polysaccharide to the SBA assay mixtures (data not shown) demonstrating that the addition of polysaccharide A inhibited killing of serogroup A bacteria while addition of polysaccharide B inhibited killing of serogroup B bacteria. 


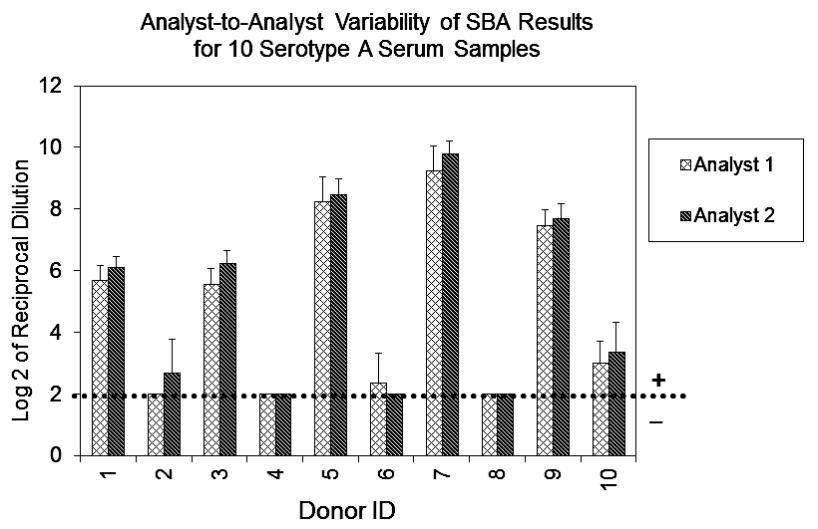

(a)

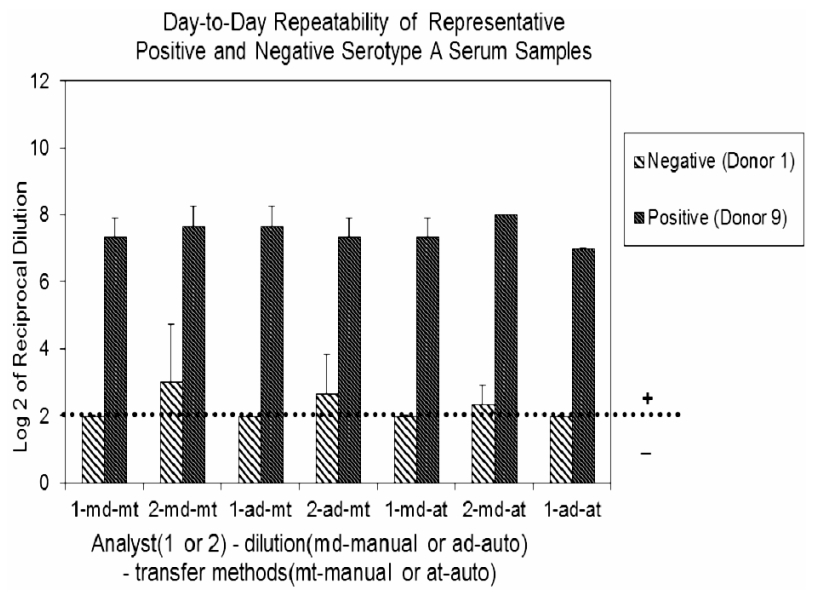

(b)

Figure 1. (a) Assay Repeatability: each vertical bar represents the mean SBA titer result of 9 repetitions for 10 sera against serogroup A polysaccharide by analyst 1 or 2, applying two dilution/ transfer methods over three days. Error bars indicate standard deviation. No error bar indicates all replicates gave identical results. (b) Assay Precision: overall day-to-day variability of ten representative serogroup A negative (Donor 1) and positive (Donor 9) sera, performed by two analysts identified as 1 or 2 . Dilution methods evaluated were manual (md) or automated (ad) and transfer methods were manual (mt) or automated (at).

\section{Discussion}

This study showed the improvement of the method by automation, and how those improvements did not impact the outcome of the results compared to manual execution. It demonstrated that the method of dilution or transfer affects, only minimally, the variability of SBA assays performed in a vacuum plate method. The use of automated dilution and transfer shows the least effect on performance while the manual dilution and manual transfermethods appear to add more variability.

Standardization and validation of the high-throughput Serum Bactericidal Assay are essential for comparisons of functional activity of antibody responses for vaccine de-

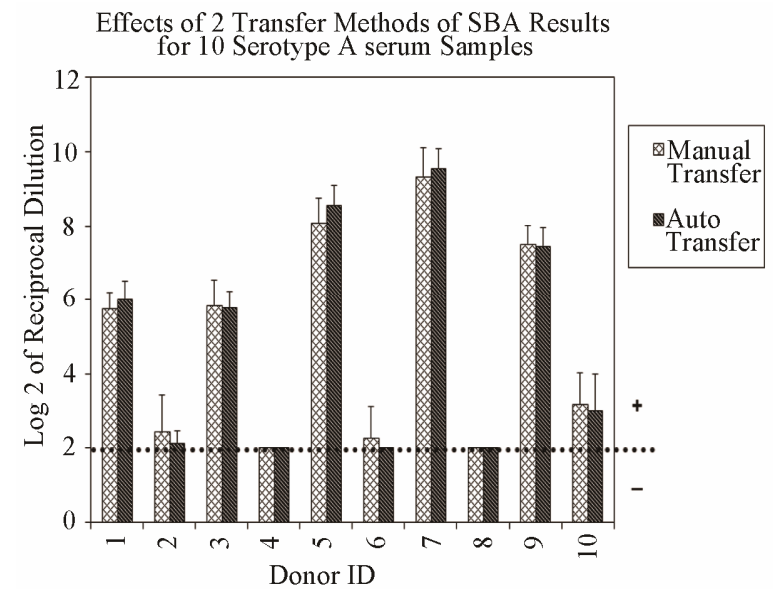

(a)

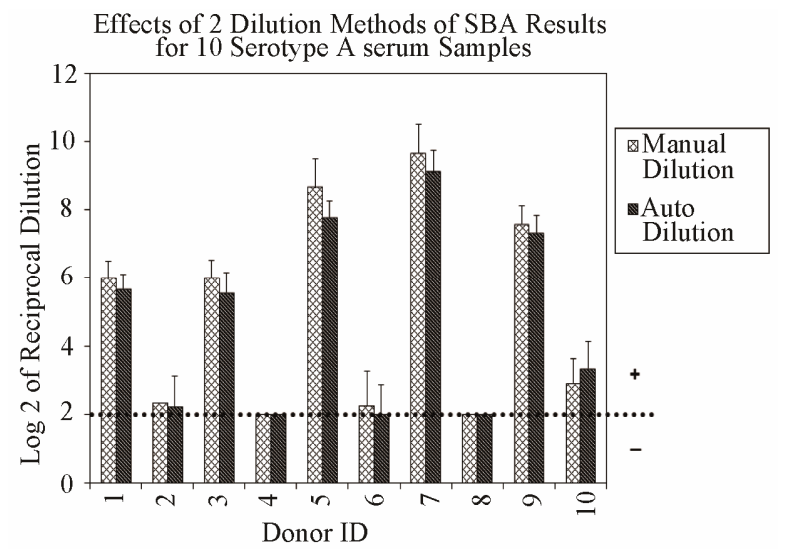

(b)

Figure 2. Assay ruggedness: each vertical bar represents the mean of six repetitions of two different dilution and transfer methods. Error bars indicate standard deviation. No error bar indicates all replicates gave identical results. (a) Effects of two transfer methods: manual transfer using multi-channel pipet and automated transfer using the RapidPlate transfer system from Qiagen. (b) Effects of two dilution methods: manual method using multi-channel pipet and automated dilution using a SerialMate instrument from Matrix Technologies Corp.

velopment. The complex nature of bioassays increases the difficulties of performing validations. This type of bioassay is time consuming and highly variable, with inherent levels of imprecision (\%RSD) occasionally as high as 50 percent [5]. The validation described here evaluated the repeatability, and precision and ruggedness of a high-throughput SBA assay. The study also examined the variability between analysts, how different methods used for dilution and transfer affect the assay performance, and how the performance affects the final outcome of the results.

Not unexpectedly, the use of a manual dilution and transfer method resulted in higher variability indicated by a higher $\%$ RSD. This variability is most likely a result of 
differences in analyst performance. The use of automated dilution and transfer equipment was predicted to reduce assay variability. The results did indeed indicate higher analyst-to-analyst variability when manual methods were used, $(\% \mathrm{RSD}=47 \%$ - $54 \%$, compared to $\% \mathrm{RSD}=34 \%$ $37 \%$ using automated methods).

In summary, we have demonstrated that the high-throughput SBA assay described here and performed independently by different analysts, over several replicates, produced consistent results. The additional automation of dilution and transfer methods does not negatively impact the assay performance. These further improvements to the assay originally described by Liu, et al. [4] should enable the rapid assessment of clinical responses to novel meningococcal vaccines and allow for direct comparison to existing vaccines.

\section{REFERENCES}

[1] E. C. Gotschlich, I. Goldschneider and M. S. Artenstein, "Human Immunity to the Meningococcus V. The Effect of Immunization with Meningococcal Group C Polysaccharide on the Carrier State," The Journal of Experimental Medicine, Vol. 129, No. 6, 1969, pp. 1385-1395.

doi:10.1084/jem.129.6.1385
[2] H. Keyserling, T. Papa, K. Koranyi, R. Ryall, E. Bassily, M. J. Bybel, K. Sullivan, G. Gilmet and A. Reinhardt, "Safety, Immunogenicity, and Immune Memory of a Novel Meningococcal (Groups A, C, Y, and W-135) Polysaccharide Diphtheria Toxoid Conjugate Vaccine (MCV-4) in Healthy Adolescents," Archives Pediatrics and Adolescent Medicine, Vol. 159, No. 10, 2005, pp. 907-913. doi:10.1001/archpedi.159.10.907

[3] S. E. Maslanka, L. L. Gheesling, D. E. Libutti, K. B. Donaldson, H. S. Harakeh, J. K. Dykes, F. F. Arhin, S. J. Devi, C. E. Frasch, J. C. Huang, P. Kriz-Kuzemenska, R. D. Lemmon, M. Lorange, C. C. Peeters, S. Quataert, J. Y. Tai and G. M. Carlone, "Standardization and a Multilaboratory Comparison of Neisseria meningitidis Serogroup A and C Serum Bactericidal Assays. The Multilaboratory Study Group," Clinical and Diagnostic Laboratory Immunology, Vol. 4, No. 2, 1997, pp. 156-167.

[4] X. Liu, S. Wang, L. Sendi and M. J. Caulfield, "High-Throughput Imaging of Bacterial Colonies Grown on Filter Plates with Application to Serum Bactericidal Assays," Journal of Immunological Methods, Vol. 292, No. 1-2, 2004, pp. 187-193. doi:10.1016/j.jim.2004.06.021

[5] L. E. Little, "Validation of Immunological and Biological Methods," Biopharm-the Technology \& Business of Biopharmaceuticals, Vol. 8, No. 9, 1995, pp. 36-42. 